EPJ manuscript No.

(will be inserted by the editor)

\title{
Interactions between heavy mesons and Goldstone bosons from chiral dynamics
}

\author{
Feng-Kun Guo ${ }^{1 \mathrm{a}}$, Christoph Hanhart ${ }^{1,2 \mathrm{~b}}$, and Ulf-G. Meißner ${ }^{1,2,3 \mathrm{c}}$ \\ 1 Institut für Kernphysik and Jülich and Center for Hadron Physics, Forschungszentrum Jülich, D-52425 Jülich, Germany \\ 2 Institute for Advanced Simulations, Forschungszentrum Jülich, D-52425 Jülich, Germany \\ 3 Helmholtz-Institut für Strahlen- und Kernphysik and Bethe Center for Theoretical Physics, Universität Bonn, D-53115 Bonn, Germany
}

Received: date / Revised version: date

\begin{abstract}
We calculate the S-wave scattering lengths for charmed mesons scattering off Goldstone bosons and explore their quark mass dependence using chiral perturbation theory up to next-to-leading order as well as a unitarized version of it. The quark mass dependence of all scattering lengths determined in a recent lattice calculation can be reproduced by the unitarized version. We also discuss signals of possible bound states in these observables.
\end{abstract}

PACS. 12.39.Fe Chiral Lagrangians - 13.75.Lb Meson-meson interactions - 14.40.Lb Charmed mesons

\section{Introduction}

The interaction strengths amongst hadrons are fundamental quantities of the strong interactions. There are two different approaches to analyze the low-energy scattering amplitudes in a model-independent manner. First, one can use the chiral perturbation theory (ChPT) representation of the pertinent scattering amplitude which is given in terms of a few LECs. These might either be determined directly from experiment or from full QCD calculations performed on a discretized space-time lattice. A beautiful example in this context is the accurate determination of the pionic LECs $l_{3}$ and $l_{4}$ from lattice simulations - see Refs. [1] and references therein. The second approach to address the issue of scattering lengths is the exploitation of Lüscher's formula [2]3] in the analysis of the lattice gauge theory results. It allows one to relate two-particle scattering lengths to the behaviour of certain energy levels in a finite volume. In full lattice QCD, the maximally stretched states for $\pi \pi, \pi K$ and $K K$ scattering have been systematically and accurately analyzed by the NPLQCD collaboration based on an extension of the Lüscher formula (for a review, see Ref. [4] and references therein).

In this paper we will discuss another sector, where the same program can be carried out, namely the scattering of Goldstone bosons off $D$-mesons. A recent lattice study for these systems is given in Ref. [5]. However, contrary to the $\pi \pi$ system, here an additional complication may occur, for the interaction in some channels might be sufficiently strong to show nonperturbative phenomena even on the level of hadron-hadron scattering, eventually leading to hadron bound states - the so-called hadronic molecules. A famous example of relevance here is the $D_{s 0}^{*}(2317)$ identified as a $D K$ bound state in various studies [6]7/8|9]. We will discuss to what extent non-perturbative phenomena might already be present in the channels investigated on the lattice and also point out the most interesting channels not yet studied. Note also that a detailed knowledge of Goldstone boson- $D$-meson scattering is not only of interest on its own, it is also an essential input for calculations of $D$-mesons in matter [10].

Preceding the direct lattice calculations of the Goldstone boson- $D$-meson scattering lengths, the one for the $I=1 / 2$ $D \pi$ channel (where $I$ denotes the total isospin) was extracted using the lattice calculations of the $D \pi$ scalar form factor in semileptonic $D \rightarrow \pi$ decays in Ref. [11]. The result was $M_{\pi} a=$ $0.29(4)$, i.e. $a=0.41(6) \mathrm{fm}$ (where $a$ denotes the scattering length). Using the same method, the value of the isoscalar $D K$ scattering length was found to be effectively infinite, which was interpreted as a signal of a bound state of $D K$ at threshold.

In this paper, we present a systematic study of the $\mathrm{S}$-wave scattering lengths of the Goldstone boson- $D$-meson interactions for all possible channels. In Section 2 the results in the physical world (i.e. at the physical values of the quark masses) are calculated to leading order (LO) and next-to-leading order (NLO) in the chiral expansion, as well as those obtained using unitarized scattering amplitudes. In Section 3, we extrapolate the results to unphysical quark masses using the unitarized scattering amplitudes. Some discussions and a brief summary is given in Section 4 . Some useful isospin relations are relegated to Appendix $\mathrm{A}$

\footnotetext{
${ }^{\mathrm{a}}$ Email address: f.k.guo@fz-juelich.de

b Email address: c.hanhart@fz-juelich.de

c Email address: meissner@itkp.uni-bonn.de
} 


\section{Scattering lengths at the physical quark masses}

\subsection{The chiral effective Lagrangians}

The leading order Lagrangian is just given by the kinetic and the mass term of the heavy mesons (chirally coupled to pions) [12 13.14$]$

$$
\mathcal{L}^{(1)}=\mathcal{D}_{\mu} D \mathcal{D}^{\mu} D^{\dagger}-\stackrel{\circ}{M}_{D}^{2} D D^{\dagger}
$$

with $D=\left(D^{0}, D^{+}, D_{s}^{+}\right)$collecting the charmed mesons with the chiral limit mass $\stackrel{\circ}{M}_{D}$. The covariant derivative is

$$
\begin{aligned}
\mathcal{D}_{\mu} & =\partial_{\mu}+\Gamma_{\mu}, \\
\Gamma_{\mu} & =\frac{1}{2}\left(u^{\dagger} \partial_{\mu} u+u \partial_{\mu} u^{\dagger}\right),
\end{aligned}
$$

where

$$
U=\exp \left(\frac{\sqrt{2} i \phi}{F}\right), \quad u^{2}=U
$$

with $F$ the Goldstone boson decay constant in the chiral limit (which we will identify with the pion decay constant in the following). The Goldstone bosons are collected in the matrixvalued field

$$
\phi=\left(\begin{array}{ccc}
\frac{1}{\sqrt{2}} \pi^{0}+\frac{1}{\sqrt{6}} \eta & \pi^{+} & K^{+} \\
\pi^{-} & -\frac{1}{\sqrt{2}} \pi^{0}+\frac{1}{\sqrt{6}} \eta & K^{0} \\
K^{-} & \bar{K}^{0} & -\frac{2}{\sqrt{6}} \eta
\end{array}\right) .
$$

Since we are only interested in the scattering lengths with definite isospin, we only consider the strong interaction part of the NLO chiral Lagrangian describing the interactions of the pseudoscalar charmed mesons with the Goldstone bosons, which reads [15]

$$
\begin{aligned}
\mathcal{L}_{\text {str. }}^{(2)} & =D\left(-h_{0}\left\langle\chi_{+}\right\rangle-h_{1} \tilde{\chi}_{+}+h_{2}\left\langle u_{\mu} u^{\mu}\right\rangle-h_{3} u_{\mu} u^{\mu}\right) \bar{D} \\
& +\mathcal{D}_{\mu} D\left(h_{4}\left\langle u^{\mu} u^{\nu}\right\rangle-h_{5}\left\{u^{\mu}, u^{\nu}\right\}-h_{6}\left[u^{\mu}, u^{\nu}\right]\right) \mathcal{D}_{\nu} \bar{D}
\end{aligned}
$$

where

$$
\begin{aligned}
& \chi_{+}=u^{\dagger} \chi u^{\dagger}+u \chi u, \\
& \tilde{\chi}_{+}=\chi_{+}-\frac{1}{3}\left\langle\chi_{+}\right\rangle, \\
& u_{\mu}=i u^{\dagger} \mathcal{D}_{\mu} U u^{\dagger} .
\end{aligned}
$$

The quark mass matrix is diagonal

$$
\chi=2 B \cdot \operatorname{diag}\left(m_{u}, m_{d}, m_{s}\right),
$$

in terms of $B=|\langle 0|\bar{q} q| 0\rangle| / F^{2}$. The unknown coefficients $h_{i}(i=$ $0, \ldots, 6)$ are the pertinent LECs. At $\mathcal{O}\left(p^{2}\right), h_{1}$ can be determined from the mass differences among the $D$-mesons as $h_{1}=$ 0.42 [15]. In the following, we choose to drop the terms with one more flavor trace in the NLO Lagrangian, i.e. the $h_{0}, h_{2}$ and $h_{4}$ terms, which are suppressed in the large $N_{C}$-limit of
QCD [16 17]. The $h_{6}$ term is also dropped since it is suppressed by one order due to the commutator structure. We are therefore left with only two free, active parameters, namely $h_{3}$ and $h_{5}$. It can be shown that the contributions of the $h_{5}$ and $h_{3}$ terms to $\mathrm{S}$-wave amplitudes differ only at $\mathcal{O}\left(p / m_{D}\right)$. Therefore a variation of $h_{3}$ within its natural bounds for a given $h_{5}$ provides an estimate for higher order contributions [15].

\subsection{Numerical results for the scattering lengths}

We first consider the perturbative chiral expansion of the scattering amplitude, $T=T^{(1)}+T^{(2)}+\ldots$, where the superscript denotes the chiral dimension. We work here to NLO, that is chiral dimension two. In that case, the scattering amplitudes are real and can be written as

$$
\begin{aligned}
T(s, t, u) & =T^{(1)}(s, t, u)+T^{(2)}(s, t, u) \\
& =\frac{C_{0}}{4 F^{2}}(s-u)+\frac{2 C_{1}}{3 F^{2}} h_{1}+\frac{2 C_{35}}{F^{2}} H_{35}(s, t, u),(8)
\end{aligned}
$$

with

$$
H_{35}(s, t, u)=h_{3} p_{2} \cdot p_{4}+h_{5}\left(p_{1} \cdot p_{2} p_{3} \cdot p_{4}+p_{1} \cdot p_{4} p_{2} \cdot p_{3}\right) \text {. }
$$

The coefficients in all the amplitudes for the Goldstone boson$D$-meson scattering are given in Table 1 The NLO S-wave elastic scattering amplitudes at threshold of the participating particles can be cast into the form

$$
\begin{gathered}
T_{\mathrm{thr}}=\frac{1}{F^{2}}\left[C_{0} M_{1} M_{2}+\frac{2 C_{1}}{3} h_{1}+2 C_{35}\left(h_{3} M_{2}^{2}\right.\right. \\
\left.\left.+2 h_{5} M_{1}^{2} M_{2}^{2}\right)\right],
\end{gathered}
$$

with $M_{1}$ and $M_{2}$ denoting the masses of the scattered heavy and light mesons, respectively. Note that from Table 1 one can see that the most attractive interaction occurs in the $(S, I)=$ $(1,0) D K$ channel, where $S(I)$ denotes the total strangeness (isospin) of the two-meson system. This is the reason why in this channel the $D_{s 0}^{*}(2317)$ was generated dynamically in many previous works [6/7/89].

The S-wave scattering length parameterizes the scattering amplitude at threshold

$$
a_{0}=-\frac{1}{8 \pi\left(M_{1}+M_{2}\right)} T_{\mathrm{thr}} .
$$

Up to the order we are working, $F$ can be replaced by the physical pion decay constant $F_{\pi}=92.4 \mathrm{MeV}$. We take the physical masses for all the mesons, i.e., $M_{\pi}=138 \mathrm{MeV}, M_{K}=$ $496 \mathrm{MeV}, M_{\eta}=548 \mathrm{MeV}, M_{D}=1867 \mathrm{MeV}$, and $M_{D_{s}}=$ $1968 \mathrm{MeV}$ [18]. The results for the S-wave scattering lengths at $\mathrm{LO}$ are given in the third column of Table 2 .

To work out the results to NLO, we take the dimensionless low-energy constant $h_{5}^{\prime} \equiv h_{5} M_{D^{0}}^{2}$ to be in the natural range of $[-1,1]$ as in Ref. [15] Correspondingly, $h_{3}$ is determined

\footnotetext{
${ }^{1}$ There is a typo in Ref. [15]. There $h_{5}^{\prime}$ was written as $h_{5} / M_{D^{0}}^{2}$ while the correct one should be $h_{5} M_{D^{0}}^{2}$.
} 


\begin{tabular}{|ll|rlr|}
\hline \hline$(S, I)$ & Channel & \multicolumn{1}{|c|}{$C_{0}$} & $C_{1}$ & $C_{35}$ \\
\hline$(-1,0)$ & $D \bar{K} \rightarrow D \bar{K}$ & -1 & $5 M_{K}^{2}$ & -1 \\
$(-1,1)$ & $D \bar{K} \rightarrow D \bar{K}$ & 1 & $-M_{K}^{2}$ & 1 \\
$\left(0, \frac{1}{2}\right)$ & $D \pi \rightarrow D \pi$ & -2 & $-M_{\pi}^{2}$ & 1 \\
& $D \eta \rightarrow D \eta$ & 0 & $2 M_{\eta}^{2}-M_{\pi}^{2}$ & $\frac{1}{3}$ \\
& $D_{s} \bar{K} \rightarrow D_{s} \bar{K}$ & -1 & $-M_{K}^{2}$ & 1 \\
& $D \eta \rightarrow D \pi$ & 0 & $-3 M_{\pi}^{2}$ & 1 \\
& $D_{s} \bar{K} \rightarrow D \pi$ & $-\frac{\sqrt{6}}{2}$ & $-\frac{3 \sqrt{6}}{4}\left(M_{K}^{2}+M_{\pi}^{2}\right)$ & $\frac{\sqrt{6}}{2}$ \\
& $D_{s} \bar{K} \rightarrow D \eta$ & $-\frac{\sqrt{6}}{2}$ & $\frac{\sqrt{6}}{4}\left(5 M_{K}^{2}-3 M_{\pi}^{2}\right)$ & $-\frac{\sqrt{6}}{6}$ \\
$\left(0, \frac{3}{2}\right)$ & $D \pi \rightarrow D \pi$ & 1 & $-M_{\pi}^{2}$ & 1 \\
$(1,0)$ & $D K \rightarrow D K$ & -2 & $-4 M_{K}^{2}$ & 2 \\
& $D_{s} \eta \rightarrow D_{s} \eta$ & 0 & $-2\left(2 M_{\eta}^{2}-M_{\pi}^{2}\right)$ & $\frac{4}{3}$ \\
& $D_{s} \eta \rightarrow D K$ & $-\sqrt{3}$ & $-\frac{\sqrt{3}}{2}\left(5 M_{K}^{2}-3 M_{\pi}^{2}\right)$ & $\frac{\sqrt{3}}{3}$ \\
$(1,1)$ & $D_{s} \pi \rightarrow D_{s} \pi$ & 0 & $2 M_{\pi}^{2}$ & 0 \\
& $D K \rightarrow D K$ & 0 & $2 M_{K}^{2}$ & 0 \\
& $D K \rightarrow D_{s} \pi$ & 1 & $-\frac{3}{2}\left(M_{K}^{2}+M_{\pi}^{2}\right)$ & 1 \\
$\left(2, \frac{1}{2}\right)$ & $D_{s} K \rightarrow D_{s} K$ & 1 & $-M_{K}^{2}$ & 1 \\
\hline \hline
\end{tabular}

Table 1. The coefficients in the scattering amplitudes. Here, $S(I)$ denotes the total strangeness (isospin) of the two-meson system.

\begin{tabular}{|ll|rccc|c|}
\hline \hline$(S, I)$ & Channel & LO & NLO & UChPT & CUChPT & Lattice [5] \\
\hline$(-1,0)$ & $D \bar{K} \rightarrow D \bar{K}$ & 0.36 & $0.31(2)$ & $0.96(20)$ & & \\
$(-1,1)$ & $D \bar{K} \rightarrow D \bar{K}$ & -0.36 & $-0.41(2)$ & $-0.22(2)$ & & $-0.23(4)$ \\
$\left(0, \frac{1}{2}\right)$ & $D \pi \rightarrow D \pi$ & 0.24 & $0.23(0)$ & $0.36(1)$ & $0.35(1)$ & \\
& $D \eta \rightarrow D \eta$ & 0 & $-0.09(1)$ & $-0.08(1)$ & $0.19(9)+i 0.02(2)$ & \\
& $D_{s} \bar{K} \rightarrow D_{s} \bar{K}$ & 0.36 & $0.31(6)$ & $1.10(57)$ & $-0.60(53)+i 0.77(15)$ & \\
$\left(0, \frac{3}{2}\right)$ & $D \pi \rightarrow D \pi$ & -0.12 & $-0.12(0)$ & $-0.10(1)$ & & $-0.16(4)$ \\
$(1,0)$ & $D K \rightarrow D K$ & 0.72 & $0.67(4)$ & $-1.47(20)$ & $-0.93(5)$ & \\
& $D_{s} \eta \rightarrow D_{s} \eta$ & 0 & $0.00(10)$ & $0.02(10)$ & $-0.33(4)+i 0.05(1)$ & \\
$(1,1)$ & $D_{s} \pi \rightarrow D_{s} \pi$ & 0 & -0.005 & -0.005 & $-0.0003(4)$ & $0.00(1)$ \\
$\left(2, \frac{1}{2}\right)$ & $D_{s} K \rightarrow D_{s} K$ & -0.36 & $-0.41(6)$ & $-0.23(5)$ & & $-0.31(2)$ \\
\hline \hline
\end{tabular}

Table 2. The S-wave scattering lengths from calculations at LO and NLO (units are fm). The results using unitarized amplitudes are also given in the two columns denoted by UChPT and CUChPT, representing one-channel and coupled-channel unitarized chiral perturbation theory, respectively.

from fitting to the mass of the $D_{s 0}^{*}(2317)$ in the full calculation, to be described below. This leads to $h_{3}=-1.479$ for $h_{5}^{\prime}=1$, and $h_{3}=2.315$ for $h_{5}^{\prime}=-1$. The results for the $\mathrm{S}$-wave scattering lengths using these input parameters also for the perturbative calculation to NLO are given in the fourth column of Table 2 with the uncertainties from the lack of knowledge of $h_{3}$ and $h_{5}^{\prime}$. We use these uncertainties as an estimate for possible higher order corrections. A comparison of the NLO results with the LO ones shows a good convergence of the chiral expansion, especially for channels where only $D$-mesons and pions are involved. Even in the channels with kaons or etas, no dramatic NLO corrections are found.

The above calculations are performed with perturbation theory up to a given order. However, a perturbative expansion to a finite order can never be reliable, if there is a bound state, a virtual state, or a resonance near by. All of these are non- perturbative phenomena, and the presence of such kind of state would modify the results from perturbation theory significantly. Therefore, we unitarize the amplitudes obtained from ChPT according to the method of Ref. [19] (called UChPT in the following. An early review on this method is [20]). The unitarized amplitude is then given by the following resummation

$$
T(s)=V(s)[1-G(s) \cdot V(s)]^{-1},
$$

with $V(s)$ the S-wave projection of the scattering amplitude $T=T^{(1)}+T^{(2)}$ given in Eq. (8). Further, $G(s)$ is the scalar two-meson loop integral [1921] regularized by a subtraction constant $a(\mu)$ with $\mu$ denoting the scale of the dimensional regularization. The expression of the loop integral at threshold is 


\begin{tabular}{|llc|ccc|ccc|}
\hline \hline$(S, I)$ & Channel & Thr & \multicolumn{3}{|c|}{$h_{5}^{\prime}=+1$} & \multicolumn{3}{|c|}{$h_{5}^{\prime}=-1$} \\
& & & $\mathrm{Re}$ & $\mathrm{Im}$ & $\mathrm{RS}$ & $\mathrm{Re}$ & $\mathrm{Im}$ & $\mathrm{RS}$ \\
\hline$(-1,0)$ & $D \bar{K} \rightarrow D \bar{K}$ & 2363 & 2354 & \pm 56 & $\mathrm{II}$ & 2301 & \pm 97 & $\mathrm{II}$ \\
$\left(0, \frac{1}{2}\right)$ & $D \pi \rightarrow D \pi$ & 2005 & 2098 & \pm 124 & $\mathrm{II}$ & 2102 & \pm 106 & $\mathrm{II}$ \\
& $D_{s} \bar{K} \rightarrow D_{s} \bar{K}$ & 2464 & 2286 & \pm 54 & $\mathrm{II}$ & 2354 & 0 & $\mathrm{II}$ \\
& & & & & & 2431 & 0 & $\mathrm{II}$ \\
$(1,0)$ & $D K \rightarrow D K$ & 2363 & 2343 & 0 & $\mathrm{I}$ & 2337 & 0 & $\mathrm{I}$ \\
\hline \hline
\end{tabular}

Table 3. Real parts, imaginary parts and Riemann sheet (RS) of the pole positions for the one-channel calculations for two parameter sets. All masses/energies are given in $\mathrm{MeV}$.

\begin{tabular}{|l|ccc|ccc|}
\hline \hline$(S, I)$ & \multicolumn{3}{|c|}{$h_{5}^{\prime}=+1$} & \multicolumn{3}{|c|}{$h_{5}^{\prime}=-1$} \\
& $\mathrm{Re}$ & $\mathrm{Im}$ & $\mathrm{RS}$ & $\mathrm{Re}$ & $\mathrm{Im}$ & $\mathrm{RS}$ \\
\hline$\left(0, \frac{1}{2}\right)$ & 2107 & \pm 123 & II & 2107 & \pm 105 & II \\
& 2452 & \pm 17 & III & 2519 & \pm 69 & III \\
$\left(0, \frac{1}{2}\right) \quad\left(V_{i i}=0\right)$ & 2466 & \pm 24 & III & 2388 & \pm 49 & III \\
$(1,0)$ & 2318 & 0 & I & 2318 & 0 & I \\
$(1,1)$ & 2309 & \pm 111 & III & 2283 & \pm 196 & III \\
\hline \hline
\end{tabular}

Table 4. Positions of poles with the largest impact on physical observables from the coupled channel calculations for two parameter sets. $V_{i i}=0$ denotes the results for a calculation where the diagonal interactions were switched off. Here the second (third) Riemann sheet for the $(0,1 / 2)$ case is defined by $\operatorname{Im}\left(q_{D \pi}\right)<0, \operatorname{Im}\left(q_{D \eta}\right)>0$, and $\operatorname{Im}\left(q_{D_{s} \bar{K}}\right)>0\left(\operatorname{Im}\left(q_{D \pi}\right)<0, \operatorname{Im}\left(q_{D \eta}\right)<0\right.$, and $\left.\operatorname{Im}\left(q_{D_{s} \bar{K}}\right)>0\right)$, and that for the $(1,1)$ case is defined by $\operatorname{Im}\left(q_{D_{s} \pi}\right)<0$, and $\operatorname{Im}\left(q_{D K}\right)>0\left(\operatorname{Im}\left(q_{D_{s} \pi}\right)<0\right.$, and $\left.\operatorname{Im}\left(q_{D K}\right)<0\right)$. All masses/energies are given in MeV.

rather simple

$$
\begin{aligned}
G\left(s_{\mathrm{thr}}\right)= & \frac{1}{16 \pi^{2}}\left[a(\mu)+\frac{1}{M_{1}+M_{2}}\right. \\
& \left.\times\left(M_{1} \ln \frac{M_{1}^{2}}{\mu^{2}}+M_{2} \ln \frac{M_{2}^{2}}{\mu^{2}}\right)\right] .
\end{aligned}
$$

We will use the subtraction constant determined in Ref. [15], i.e. $a(1 \mathrm{GeV})=-1.846$. Then the scattering lengths with the unitarized amplitude for each channel can be obtained easily, and the results are given in the column denoted by UChPT in Table 2 For the channels with repulsive interactions, i.e. those with negative scattering lengths at LO and NLO in the convention used here, the correction from the resummation is not dramatic. It is several percent for the channels without kaons, and $50 \%$ for the channels with kaons at most. For the channels with attractive interactions, i.e. those with positive scattering lengths at LO and NLO, the smallest correction is already $50 \%$ for the $(0,1 / 2) D \pi$ channel. Both the scattering lengths of the $(-1,0)$ $D \bar{K}$ and $(0,1 / 2) D_{s} \bar{K}$ channels change from about $0.3 \mathrm{fm}$ to about $1 \mathrm{fm}$, and that of the $(1,0) D K$ even changes the sign. These dramatic changes can be understood on the basis of the singularity structure of the unitarized amplitudes.

At this point some general remarks are in order. Imagine some energy-independent potential that provides an attractive force between two particles. As the strength of the force is increased, eventually a virtual state appears in the unitarized scattering amplitude. A virtual state is a singularity below threshold on the real axis on the second sheet of the complex $s$-plane of the S-matrix. As the interaction strength is further increased the pole moves closer and closer to the threshold. At the same time the scattering length grows to become positive infinity when the pole hits the threshold. This situation is almost realized in $n n$ scattering near threshold which shows an exceptionally large scattering length of about $18 \mathrm{fm}$. If the strength of the interaction is increased even further, the pole jumps on the physical sheet - the state becomes a bound state. Correspondingly the scattering length changes sign and starts to increase from negative infinity as the pole moves away from the threshold - this is the case that applies to the deuteron channel of nucleon-nucleon scattering, where the scattering length is about $-5 \mathrm{fm}$ (Note that the sign conventions in nucleonnucleon scattering are often different to those used here). These scenarios are discussed in various textbooks, see e.g. Refs. [22 23]. For energy-dependent interactions one may even start from a resonance, which corresponds to two poles in the second Riemann sheet, both located as mirror images with respect to reflections on the real axis. This is illustrated as the points A and $\mathrm{A}^{\prime}$ in Fig. 1. Then an increase of the interaction strength will let the magnitude of both the real part and the imaginary part decrease and will eventually lead to poles located below the elastic threshold, but still with non-vanishing imaginary parts ( $\mathrm{B}$ and $\mathrm{B}$ ' in the figure). A further increase in the strength of the potential might convert the two resonance poles into two virtual states ( $\mathrm{C}$ and $\mathrm{C}$ ' in the figure). As the strength parameter gets increased even further, one pole moves towards the threshold to eventually become a bound state while the other one moves further away from the physical regime - this behaviour was e.g. reported in Ref. [24] for the case of the light scalar meson $f_{0}(600)$, where the interaction strength was varied by a variation of the pion mass and was also observed in phenomenological studies [25]. We checked that indeed the mentioned types 


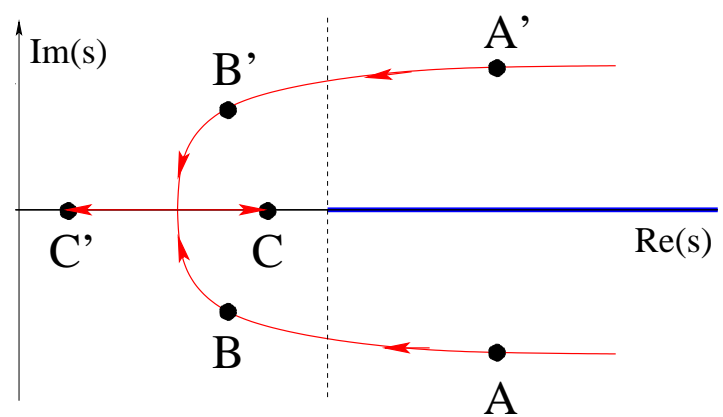

Fig. 1. Sketch of typical trajectories of $S$-matrix poles in the second Riemann sheet of the complex $s$-plane for energy-dependent potentials when some strength parameter is changed. See text for meaning of the labels. The dashed vertical line indicates the position of the elastic scattering threshold and the thick horizontal line the resulting unitarity cut.

of poles appear by the unitarization. As can be read off Table 3 , showing the pole positions for the single channel calculations, for $h_{5}^{\prime}=+1$ in the $D_{s} \bar{K}$ channel there appears a pair of resonance poles below the $D_{s} \bar{K}$ threshold. For the other parameter set displayed in the table $\left(h_{5}^{\prime}=-1\right)$ those changed into two virtual states. For the full calculation with coupled channels for both parameter sets there are only resonance poles. In addition there are pairs of resonance poles in the second Riemann sheet for the channels $(0,1 / 2) D \pi$ and $(-1,0) D \bar{K}$. The former one was found already previously in Ref. [7]. Note also that our findings for the scattering length in that channel are consistent with those of Ref. [11] within the theoretical uncertainty. In the $(1,0) D K$ even a bound state appears. As a consequence the scattering length changes its sign. In Refs. [26] in was shown that for bound states near a threshold one may write for the scattering length

$$
a=-2\left(\frac{1-Z}{2-Z}\right) \frac{1}{\sqrt{2 \mu \epsilon}}(1+\mathcal{O}(\sqrt{2 \mu \epsilon} / \beta)) .
$$

Applied to the $D_{s 0}^{*}(2317)$ we have $\epsilon=M_{D}+M_{K}-M_{D_{s 0}^{*}(2317)}$ for the binding energy, $\mu=M_{D} M_{K} /\left(M_{D}+M_{K}\right)$ for the reduced mass, and $1 / \beta$, the range of forces, may be estimated via $\beta \sim m_{\rho}$. The quantity $Z$, which can be identified with the wave function renormalization constant, is a measure of the molecular component of the state, with $Z=1(Z=0)$ for a pure elementary (molecular) state. Taking $Z=0$, the above equation gives $a_{D K \rightarrow D K}^{(1,0)}=-1.05 \mathrm{fm}$, which is close to the value listed in Table 2. Thus, would this value be extracted from lattice simulations in the future, it would be a direct proof for the molecular nature of the $D_{s 0}^{*}(2317)$.

For $(S, I)=(0,1 / 2),(1,0)$ and $(1,1)$, there are more channels with the same quantum numbers, and the unitarization should include also the coupled-channel effect (called CUChPT for simplicity). The off-diagonal interactions can induce an imaginary part to the scattering length for those channels coupling to a channel with a lower threshold. The results with coupled-channel unitarization are given in the column denoted by CUChPT in Table 2. From the amplitude coefficients for $(S, I)=(0,1 / 2)$ in Table 1, one finds the off-diagonal interactions for the $D_{s} \bar{K}$ to $D \pi$ and $D \eta$ are large. As can be seen from the line labled by $V_{i i}=0$ in Table 4 they alone can already produce poles. For the full calculation their effect is to move the poles on the second Riemann sheet of the one-channel $D_{s} \bar{K} \rightarrow D_{s} \bar{K}$ amplitude to a pole in the relative first Riemann sheet of the $D_{s} \bar{K}$ channel (that is the Riemann sheet with positive imaginary part of the center-of-mass system $(\mathrm{cms})$ three-momentum in this channel and negative cms three-momentum in the other two channels - for the full calculation this sheet is labled as III, for by convention the first sheet is related to the channel with the lightest threshold). The appearance of the pole on that physical sheet again leads to a change in sign of the real parts of the scattering lengths for both the $D \eta$ and $D_{s} \bar{K}$ channels. For $(S, I)=(1,1)$, the LO diagonal interactions vanish, and the NLO ones are repulsive. The off-diagonal interaction however produces a pole which is shown in the last line in Table 4 Thus the pole is purely a coupled-channel effect. Because of the pole the imaginary part of the scattering length in the $(1,1) D K$ channel becomes much larger than its real part, a direct reflection of the coupledchannel effect.

There are no experimental data for the scattering of Goldstone bosons off $D$-mesons available. However, for four of the channels discussed above results of lattice gauge theory calculations were recently published [5]. These lattice data have to be taken with a grain of salt as they have been obtained for a single lattice spacing and volume, thus leaving room for sizeable systematic discretization effects. The resulting scattering lengths are also shown in Table 2. To illustrate the level of agreement for the LO, NLO, and UChPT calculations with the lattice data the results for three of the channels are also shown in Fig. 2 (so far lattice data exist only for channels without channel coupling). For the fourth channel investigated on the lattice the scattering length is very small as it is for all the different calculations we performed. Instead of this channel, in the figure we show the channel $(-1,0) D \bar{K} \rightarrow D \bar{K}$, which could be easily investigated on the lattice with the same tools already employed in Ref. [5]. The first and important observation is that the NLO corrections are generally small - the series appears to be well behaved. In addition, the figure shows that for two of the channels, $\left(0, \frac{3}{2}\right) D \pi \rightarrow D \pi$ and $\left(2, \frac{1}{2}\right) D_{s} K \rightarrow D_{s} K$, the agreement of the calculations with the lattice results is of similar quality for all three schemes, however, for the channel $(-1,1) D \bar{K} \rightarrow D \bar{K}$ an agreement with the lattice results is achieved only after unitarization. This is a priori unexpected, since the interaction in this channel is repulsive. However, a repulsive interaction iterated to even orders gives an attractive 

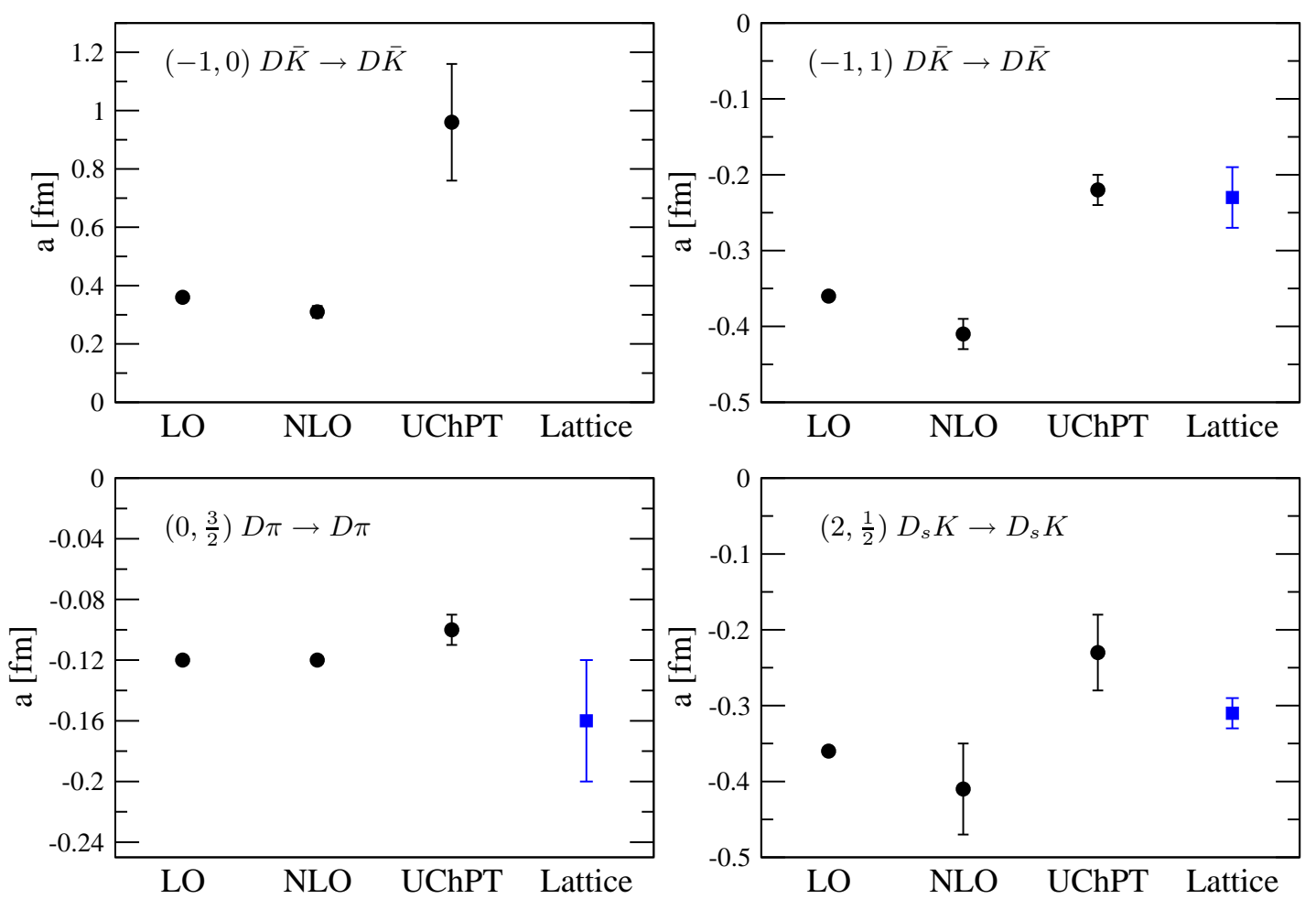

Fig. 2. Comparison of the scattering lengths in four channels with the lattice data. We give the results for LO, NLO and unitarized ChPT.

interaction and consequently by the unitarization the repulsion gets weakened - the scattering length gets smaller in magnitude. We checked that in our calculation the leading loop, formally a next-to-next-to-leading order contribution, already gives the bulk of the effect. Thus here the lattice results clearly show the trace of a higher order hadron-hadron interaction. As the first panel of the figure shows, we predict an even more dramatic effect in the channel $(-1,0) D \bar{K} \rightarrow D \bar{K}$. As discussed above, here the interaction is attractive and the large change in the scattering length is the consequence of the appearance of a resonance state in the scattering matrix - clearly a nonperturbative phenomenon. It would therefore be of high theoretical interest to have lattice results for this channel as well.

Ideally lattice simulations would be available for all channels calculated. As can be read off Table 2, in some channels the effect from the unitarization is very large while in others it is quite moderate. All this is calculated without any free parameter. Therefore a comparison of the lattice results to those from our calculation would provide a very non-trivial test of the presence of non-perturbative hadron-hadron interactions.

\section{Chiral extrapolation}

The lattice results used here are calculated with finite lattice spacing and at unphysical masses of the $u$ and $d$ quarks the masses of the strange and charm quarks are chosen at their physical values. In the lattice calculation of the charmed mesonlight hadron interactions [5], the lattice spacing is $b=0.12 \mathrm{fm}$. The mass of the $s$ quark is $80 \mathrm{MeV}$, which is consistent with its physical mass, and four values are chosen for the masses of the $u$ and $d$ quarks which are $11 \mathrm{MeV}, 16 \mathrm{MeV}, 32 \mathrm{MeV}$ and $48 \mathrm{MeV}$, respectively. Evidently, these are larger than the physical $u(d)$ quark mass. To compare the lattice results with the physical quantities, one needs to do extrapolations. Here we only discuss the chiral extrapolation of the results from the unphysical quark masses to the physical quark mass. In Ref. [5], the authors parameterize the scattering lengths using the first non-vanishing term in the chiral expansion. They used $c_{1}+c_{2} M_{\pi} / F_{\pi}$ to extrapolate the $I=3 / 2 D \pi$ scattering length, and $c_{1}+c_{2} M_{\pi}^{2} / F_{\pi}^{2}$ to extrapolate the $D_{s} \pi(K)$ and the $I=1$ $D \bar{K} \_$scattering lengths. The values they got are shown in the last column of Table 2 and Fig. 2 .

The lattice results for the scattering lengths at unphysical quark masses are given at several chosen values of $M_{\pi} / F_{\pi}$, which were taken from Ref. [28]. Therefore, we take the corresponding values of unphysical $M_{\pi}$ from the same paper for the lattice data points shown in Fig. 3. Although the masses of the charmed mesons have been expanded to one loop order [29], for our purpose we only need to work to $\mathcal{O}\left(M_{\pi}^{2}\right)$. From the Lagrangian given in Eq. (5), modulo an overall unmeasurable term proportional to $\left\langle\chi_{+}\right\rangle$which can be absorbed into the large $-N_{c}$ suppressed $h_{0}$ term, one gets for the NLO correction to the mass squares of the charmed mesons

$$
\delta M_{D}^{2}=4 h_{1} B \hat{m}, \delta M_{D_{s}}^{2}=4 h_{1} B m_{s},
$$

where $\hat{m}=\left(m_{u}+m_{d}\right) / 2$. Since the lattice calculations [5] use a value for $m_{s}$ which is close to the physical $s$ quark mass, we will use the physical mass of the $D_{s}$. From the first identity in

\footnotetext{
${ }^{2}$ In Ref. [5] this channel was called $D K$. However, the channel calculated is $[\bar{c} u][\bar{s} u]$, i.e. $\bar{D}^{0} K^{+}[27]$.
} 

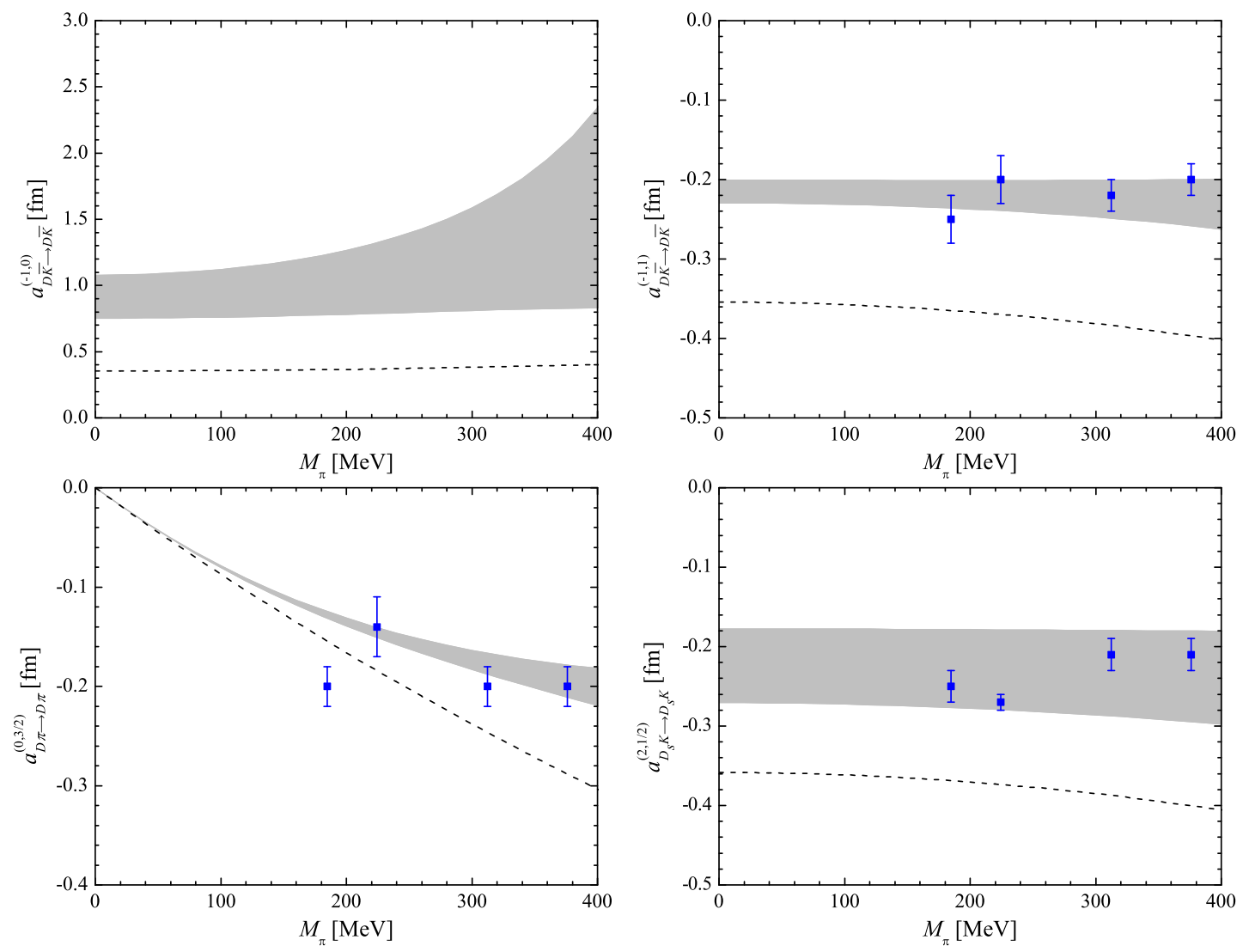

Fig. 3. Chiral extrapolation for the leading order results (dashed lines) and the full UChPT calculation (bands) compared with the lattice data.

Eq. (15), the mass of the $D$ meson up to NLO can be written as

$$
M_{D}=\stackrel{\circ}{M}_{D}+h_{1} \frac{M_{\pi}^{2}}{\stackrel{\circ}{M}_{D}}
$$

using $M_{\pi}^{2}=2 B \hat{m}$. Using $M_{K}^{2}=B\left(\hat{m}+m_{s}\right)$, one gets a similar expression for the kaon mass up to $\mathcal{O}\left(M_{\pi}^{2}\right)$ as

$$
M_{K}=\stackrel{\circ}{M}{ }_{K}+\frac{M_{\pi}^{2}}{4 \stackrel{\circ}{M}_{K}},
$$

where $\stackrel{\circ}{M}_{K}$ denotes the mass of the kaon in the SU(2) chiral limit $m_{u}=m_{d}=0$, i.e. $\stackrel{\circ}{M}_{K}^{2}=B m_{s}$. With the above expansion of $M_{D}$ and $M_{K}$, one is ready to extrapolate the results given in Section 2 in the physical world to the world with unphysical quark masses. Assuming the subtraction constant to be $M_{\pi}$-independent, which is true to NLO, the chiral extrapolations of the results using UChPT for the same channels as in Fig. 2 are shown in Fig. 3 together with the lattice data. The description of the lattice data is rather good. The width of the band reflects the remaining freedom in the choice of the parameters which is taken as an estimate of higher order effects. For comparison with the dashed line we also show the result to leading order. Although influencing the magnitude of the scattering lengths in all channels, only in the $(0,3 / 2) D \pi$ channel the higher order effects also changed the pion mass dependence, since only in this channel the changes in the light quark masses are not dwarfed by the large strange quark mass.
As discussed before, in the $(0,1 / 2) D \pi$ and the $(1,0) D K$ channels, poles get generated through the unitarization which govern the low-energy physics. Their presence is made apparent in Fig. 4, which gives the quark mass dependence of the scattering lengths for these channels predicted in CUChPT. To do the calculations the mass of the $\eta$, which enters the coupledchannel calculations, was expanded as

$$
M_{\eta}=\stackrel{\circ}{M}_{\eta}+\frac{M_{\pi}^{2}}{6 \stackrel{\circ}{M_{\eta}}} .
$$

The strength of the attractive interaction in the $(0,1 / 2) D \pi$ channel is measured by $M_{\pi}$. When $M_{\pi}$ is increased to some value, the interaction becomes strong enough to form a bound state. The structure shown in the left panel of Fig. 4 is a nice illustration of the physics discussed in Subsection 2.2. In the $(1,0) D K$ channel, on the other hand, the strength of the interaction is controlled by $M_{K}$, which is changed only moderately when the $u, d$ quark masses get increased. As a result also the scattering length shows a weak $M_{\pi}$ dependence.

\section{Summary}

In this paper we calculated the scattering lengths for all the Goldstone boson- $D$-meson scattering channels using both chiral perturbation theory as well as a unitarized version of it. We 

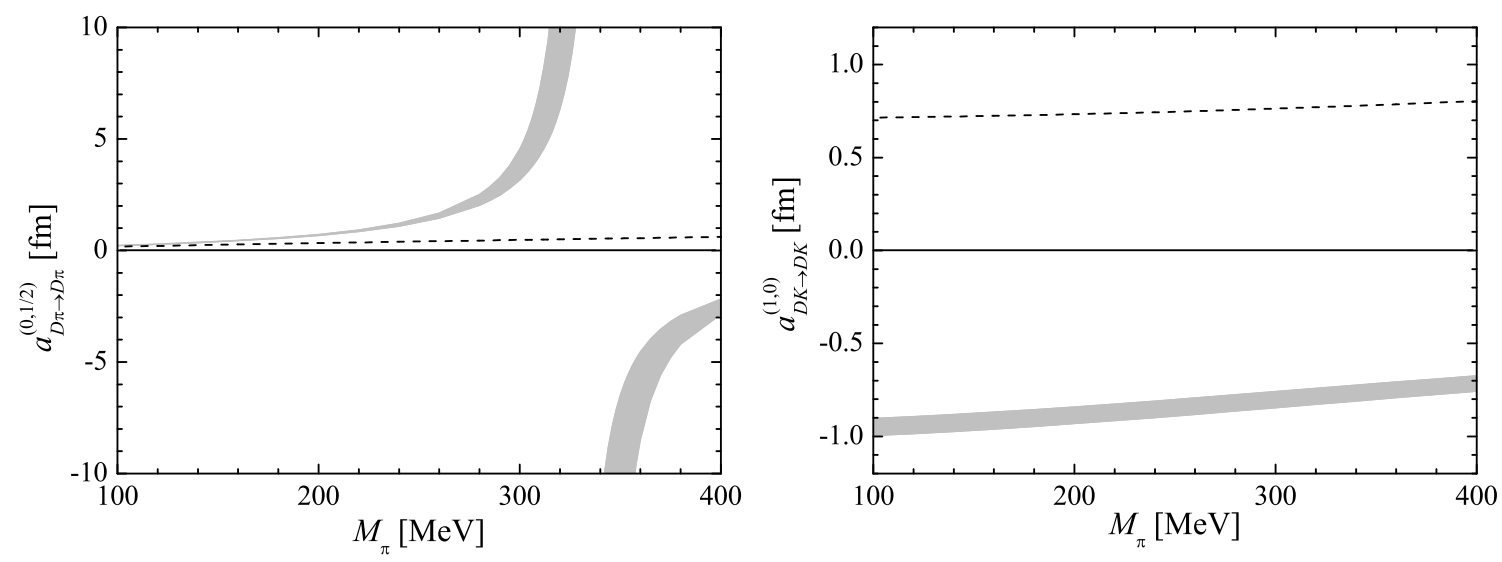

Fig. 4. Chiral extrapolation for the leading order results (dashed lines) and the full CUChPT calculation (bands) for the $(0,1 / 2) D \pi$ and the $(1,0) D K$ channels.

found that for some channels the unitarization produced new singularities in the $\mathrm{S}$-matrix, in some cases as virtual states or resonances, in some cases as bound states. One of these dynamically generated singularities can be identified with the experimentally well established $D_{s 0}^{*}(2317)$, that was identified as a hadronic molecule in various works [67889]. However, we found more non-perturbative effects and demonstrated that a determination of scattering lengths on the lattice provides an ideal tool to investigate them.

\section{Acknowledgments}

We would like to thank Liuming Liu and Kostas Orginos for useful discussions. This work is partially supported by the Helmholtz Association through funds provided to the virtual institute "Spin and strong QCD" (VH-VI-231) and by the DFG (SFB/TR 16, "Subnuclear Structure of Matter"). We also acknowledge the support of the European Community-Research Infrastructure Integrating Activity "Study of Strongly Interacting Matter" (acronym HadronPhysics2, Grant Agreement n. 227431) under the Seventh Framework Programme of EU.

\section{A Isospin relations}

In the present work, we do not consider isospin violation. In this Appendix, we give the relations between the scattering amplitudes in isospin basis and in particle basis which are useful in derivations. Before that, let us give the definition of Mandelstam variables and the phase convention for the isospin eigenstates

$$
s=\left(p_{1}+p_{2}\right)^{2}, \quad t=\left(p_{1}-p_{3}\right)^{2}, \quad u=\left(p_{1}-p_{4}\right)^{2},
$$

and

$$
\begin{aligned}
& \left|\pi^{+}\right\rangle=-|1,+1\rangle,\left|\bar{K}^{0}\right\rangle=-\left|\frac{1}{2},+\frac{1}{2}\right\rangle, \\
& \left|D^{+}\right\rangle=-\left|\frac{1}{2},+\frac{1}{2}\right\rangle .
\end{aligned}
$$

The two numbers in $|\cdots\rangle$ on the r.h.s. are $I, I_{3}$. All the other states are defined with a positive sign. Then employing isospin symmetry and crossing symmetry, one can get the following isospin relations:

$$
\begin{aligned}
& V_{D \bar{K} \rightarrow D \bar{K}}^{(-1,1)}(s, t, u)=V_{D^{0} K^{-} \rightarrow D^{0} K^{-}}(s, t, u), \\
& V_{D \bar{K} \rightarrow D \bar{K}}^{(-1,0)}(s, t, u)=2 V_{D^{+} K^{+} \rightarrow D^{+} K^{+}}(u, t, s) \\
& -V_{D^{0} K^{-} \rightarrow D^{0} K^{-}}(s, t, u), \\
& V_{D \pi \rightarrow D \pi}^{(0,3 / 2)}(s, t, u)=V_{D^{+} \pi^{+} \rightarrow D^{+} \pi^{+}}(s, t, u), \\
& V_{D \pi \rightarrow D \pi}^{(0,1 / 2)}(s, t, u)=\frac{3}{2} V_{D^{+} \pi^{+} \rightarrow D^{+} \pi^{+}}(u, t, s) \\
& -\frac{1}{2} V_{D^{+} \pi^{+} \rightarrow D^{+} \pi^{+}}(s, t, u), \\
& V_{D \eta \rightarrow D \eta}^{(0,1 / 2)}(s, t, u)=V_{D^{+} \eta \rightarrow D^{+} \eta}(s, t, u), \\
& V_{D_{s} \bar{K} \rightarrow D_{s} \bar{K}}^{(0,1 / 2)}(s, t, u)=V_{D_{s}^{+} K^{+} \rightarrow D_{s}^{+} K^{+}}(u, t, s) \text {, } \\
& V_{D \eta \rightarrow D \pi}^{(0,1 / 2)}(s, t, u)=\sqrt{3} V_{D^{0} \eta \rightarrow D^{0} \pi^{0}}(s, t, u), \\
& V_{D_{s} \bar{K} \rightarrow D \pi}^{(0,1 / 2)}(s, t, u)=\sqrt{3} V_{D_{s}^{+} K^{-} \rightarrow D^{0} \pi^{0}}(s, t, u), \\
& V_{D_{s} \bar{K} \rightarrow D \eta}^{(0,1 / 2)}(s, t, u)=V_{D_{s}^{+} K^{-} \rightarrow D^{0} \eta}(s, t, u), \\
& V_{D K \rightarrow D K}^{(1,0)}(s, t, u)=2 V_{D^{0} K^{-} \rightarrow D^{0} K^{-}}(u, t, s) \\
& -V_{D^{+} K^{+} \rightarrow D^{+} K^{+}}(s, t, u), \\
& V_{D_{s} \eta \rightarrow D_{s} \eta}^{(1,0)}(s, t, u)=V_{D_{s}^{+} \eta \rightarrow D_{s}^{+} \eta}(s, t, u), \\
& V_{D_{s} \eta \rightarrow D K}^{(1,0)}(s, t, u)=-\sqrt{2} V_{D_{s}^{+} \eta \rightarrow D^{0} K^{+}}(s, t, u), \\
& V_{D_{s} \pi \rightarrow D_{s} \pi}^{(1,1)}(s, t, u)=V_{D_{s}^{+} \pi^{0} \rightarrow D_{s}^{+} \pi^{0}}(s, t, u), \\
& V_{D K \rightarrow D K}^{(1,1)}(s, t, u)=V_{D^{+} K^{+} \rightarrow D^{+} K^{+}}(s, t, u), \\
& V_{D K \rightarrow D_{s} \pi}^{(1,1)}(s, t, u)=\sqrt{2} V_{D_{s}^{+} K^{-} \rightarrow D^{0} \pi^{0}}(u, t, s), \\
& V_{D_{s} K \rightarrow D_{s} K}^{(2,1 / 2)}(s, t, u)=V_{D_{s}^{+} K^{+} \rightarrow D_{s}^{+} K^{+}}(s, t, u) \text {, }
\end{aligned}
$$

where the superscripts mean $(S, I)$ with $S(I)$ representing the total strangeness (isospin) of the two-meson system. 


\section{References}

1. Ph. Boucaud et al. [ETM Collaboration], Phys. Lett. B 650, 304 (2007) |arXiv:hep-lat/0701012]; M. Lin [RBC and UKQCD Collaborations], PoS LAT2006, 185 (2006) |arXiv:hep-lat/0610052]; H. Matsufuru [JLQCD Collaboration and TWQCD Collaboration], PoS LAT2007, 018 (2007) |arXiv:0710.4225 [hep-lat]].

2. M. Lüscher, Commun. Math. Phys. 105, 153 (1986).

3. M. Lüscher, Nucl. Phys. B 354, 531 (1991).

4. S. R. Beane, K. Orginos and M. J. Savage, Int. J. Mod. Phys. E 17, 1157 (2008) |arXiv:0805.4629 [hep-lat]].

5. L. Liu, H. W. Lin and K. Orginos, arXiv:0810.5412 [hep-lat].

6. E. van Beveren and G. Rupp, Phys. Rev. Lett. 91, 012003 (2003) [arXiv:hep-ph/0305035].

7. E. E. Kolomeitsev and M. F. M. Lutz, Phys. Lett. B 582, 39 (2004) [arXiv:hep-ph/0307133].

8. F. K. Guo, P. N. Shen, H. C. Chiang and R. G. Ping, Phys. Lett. B 641, 278 (2006) |arXiv:hep-ph/0603072].

9. D. Gamermann, E. Oset, D. Strottman and M. J. Vicente Vacas, Phys. Rev. D 76, 074016 (2007) [arXiv:hep-ph/0612179].

10. L. Tolos, D. Cabrera, D. Gamermann, T. Mizutani, R. Molina, E. Oset and A. Ramos, arXiv:0812.1460 [hep-ph].

11. J. M. Flynn and J. Nieves, Phys. Rev. D 75, 074024 (2007) [arXiv:hep-ph/0703047].

12. G. Burdman and J. F. Donoghue, Phys. Lett. B 280, 287 (1992).

13. M. B. Wise, Phys. Rev. D 45, 2188 (1992).

14. T. M. Yan, H. Y. Cheng, C. Y. Cheung, G. L. Lin, Y. C. Lin and H. L. Yu, Phys. Rev. D 46, 1148 (1992) [Erratum-ibid. D 55, 5851 (1997)].
15. F. K. Guo, C. Hanhart, S. Krewald and U. G. Meißner, Phys. Lett. B 666, 251 (2008) \arXiv:0806.3374[hep-ph]].

16. A. V. Manohar, arXiv:hep-ph/9802419.

17. M. F. M. Lutz and M. Soyeur, Nucl. Phys. A 813, 14 (2008) [arXiv:0710.1545 [hep-ph]]

18. C. Amsler et al. [Particle Data Group], Phys. Lett. B 667, 1 (2008).

19. J. A. Oller and U.-G. Meißner, Phys. Lett. B 500, 263 (2001) [arXiv:hep-ph/0011146].

20. J. A. Oller, E. Oset and A. Ramos, Prog. Part. Nucl. Phys. 45, 157 (2000) [arXiv:hep-ph/0002193].

21. J. A. Oller and E. Oset, Phys. Rev. D 60, 074023 (1999) [arXiv:hep-ph/9809337].

22. J. R. Taylor, Scattering theory: The quantum theory of nonrelativistic collisions, Dover Publications 2006, New York.

23. K. W. McVoy, Nucl. Phys. A 115, 481 (1968).

24. C. Hanhart, J. R. Peláez and G. Ríos, Phys. Rev. Lett. 100, 152001 (2008) [arXiv:0801.2871 [hep-ph]].

25. E. van Beveren et al., AIP Conf. Proc. 660, 353 (2003) |arXiv:hep-ph/0211411]; Phys. Rev. D 74, 037501 (2006) [arXiv:hep-ph/0509351].

26. S. Weinberg, Phys. Rev. 137, B672 (1965); V. Baru, J. Haidenbauer, C. Hanhart, Yu. Kalashnikova and A. E. Kudryavtsev, Phys. Lett. B 586, 53 (2004) |arXiv:hep-ph/0308129].

27. Private communications with Liuming Liu and Kostas Orginos.

28. A. Walker-Loud et al., arXiv:0806.4549 [hep-lat].

29. E. E. Jenkins, Nucl. Phys. B 412, 181 (1994) arXiv:hep-ph/9212295. 